\title{
Meeting George Bush versus Meeting Cinderella: The Neural Response When Telling Apart What is Real from What is Fictional in the Context of Our Reality
}

\author{
Anna Abraham, D. Yves von Cramon, and Ricarda I. Schubotz
}

\begin{abstract}
A considerable part of our lives is spent engaging in the entertaining worlds of fiction that are accessible through media such as books and television. Little is known, however, about how we are able to readily understand that fictional events are distinct from those occurring within our real world. The present functional imaging study explored the brain correlates underlying such abilities by having participants make judgments about the possibility of different scenarios involving either real or fictional characters being true, given the reality of our world. The processing of real and fictional scenarios activated a common set of regions including medial-temporal lobe structures. When the scenarios involved real people, brain regions associated with episodic memory retrieval and self-referential thinking, the an-
\end{abstract}

\section{INTRODUCTION}

We regularly encounter multiple fictional worlds in our daily lives, such as when we watch movies, read books, or play computer adventure games. Thus, narratives and storytelling seem to be an integral part of our lives (for a review on the neuropsychology of narratives, see Mar, 2004). Engaging in narratives enables us not only to derive enjoyment from being entertained (Green, Brock, \& Kaufman, 2004) or feel the comfort of exploring alternate worlds from a safe vantage point (Nell, 2002) but it also is a fundamental means by which we acquire knowledge about fictional worlds as well as our own world (Marsh, Meade, \& Roediger, 2003).

We are able to effortlessly understand and appreciate the dynamics of fictional worlds, to the extent that we occasionally find ourselves completely immersed within the happenings of these worlds (Nell, 1988) so as to be emotionally affected by them (Oatley, 2002). In fact, the extent to which one feels absorbed in a story or transported into a fictional world (Gerrig, 1993) can have a significant impact on one's beliefs and evaluations

Max Planck Institute for Human Brain and Cognitive Sciences, Leipzig, Germany terior prefrontal cortex and the precuneus/posterior cingulate, were more active. In contrast, areas along the left lateral inferior frontal gyrus, associated with semantic memory retrieval, were implicated for scenarios with fictional characters. This implies that there is a fine distinction in the manner in which conceptual information concerning real persons in contrast to fictional characters is represented. In general terms, the findings suggest that fiction relative to reality tends to be represented in more factual terms, whereas our representations of reality relative to fiction are colored by personal subjectivity. What modulates our understanding of the relative difference between reality and fiction seems to be whether such character-type information is coded in self-relevant terms or not.

(Green \& Brock, 2000). What is therefore remarkable is how easily we can, nonetheless, tell fiction apart from reality, and how rarely, if ever, healthy individuals confuse fiction with reality. ${ }^{1}$ Indeed, this ability emerges early during development (Woolley \& Wellman, 1990), and by the age of 5 , children can not only differentiate reality from fiction but can also distinguish between different fictional worlds (Skolnick \& Bloom, 2006). The neural correlates underlying our abilities to distinguish between reality and fiction are, however, as yet unknown.

When reading a novel or watching cartoons, we are drawn or transported into alternative worlds to that of our own reality. It has been recently suggested that the reason why overlapping brain regions are implicated in tasks that tap episodic memory, theory of mind, navigation, and prospection is because all these situations involve a form of "self-projection" into a possible world (Buckner \& Carroll, 2007). Self-projection refers to the ability to consider alternatives to a situation at hand and it requires a shift from one's immediate perspective to consider alternative perspectives (Buckner \& Carroll, 2007). One open question is whether this network of regions would be differentially implicated when considering alternative events involving either real or fictional protagonists. 
The purpose of the present study was to uncover the neural signature associated with evaluating reality-based scenarios that involved either real or fictional characters. To this end, we devised a number of scenarios for a functional magnetic resonance imaging (fMRI) study in which a real protagonist named Peter is involved in either informative contexts (e.g., heard about on the radio, read in a newspaper) or interactive contexts (e.g., spoke to yesterday, dined with yesterday) together with a character that was either fictional (e.g., Cinderella) or a famous real person (e.g., George Bush). The participants' task was to evaluate whether each scenario was possible or not (Figure 1) by determining whether the event it portrayed could occur given the physical reality of our world.

Within this framework, it would be, for instance, possible that Peter was informed about George Bush or Cinderella by having read something about them in a newspaper. However, although it could be the case that Peter interacted with a real person such as George Bush, it would be factually impossible that he interacted with a fictional character such as Cinderella. To ensure that participants would have to make an equal number of "yes" and "no" responses when making a decision, a question cue ("Possible?" or "Impossible?") was presented to the subjects after each scenario sentence to which they had to prepare the appropriate "yes" or "no" response (Figure 1). So, for instance, if the question cue "Possible?" followed the scenario "Peter spoke to Cinderella yesterday," the correct response would involve pressing the "no" button. If, instead, the question cue "Impossible?" followed the same scenario, the correct response would involve pressing the "yes" button.

As the results from a behavioral pilot study indicated that the conditions involving fictional characters were accompanied by longer reaction times (RTs), a demanding control condition was included so that inclusive mask contrasts of the fMRI data could be carried out. In the control condition, participants had to make judgments concerning their two response keys. During the course of the experiment, the left button press was always used to indicate "yes" as an answer to the question cue and the right button press always signaled a "no" response. In line with these response codes, the control condition statements were devised to be either true (e.g., The "yes" button is the left button) or false (e.g., The "yes" button is the right button). The trial events of the control conditions were made comparable to the experimental conditions by having one of two question cues ("True?" or "False?") follow such statements to which the participants were required to accurately respond.

Although there was a strong correspondence in the pattern of activation of the experimental conditions relative to the control condition, the results reveal a dissociation between the processing of contexts involving real versus fictional characters such that neural regions customarily involved in episodic memory retrieval and self-referential processing were more engaged when making reality-based judgments about real compared to fictional characters, whereas semantic memory retrieval areas were implicated when making reality-based judgments about fictional compared to real entities.
Figure 1. Examples of scenarios for all experimental conditions (top) and a schematic representation of the sequence of events in a trial (trial length: $8 \mathrm{sec}$ ) (bottom). Across all experimental conditions, each trial began with a fixation cross (duration: $500 \mathrm{msec}$ ), which was followed by the presentation of single sentence for $2000 \mathrm{msec}$ where a scenario was introduced. Following a delay (500 msec), a question cue was presented to which the participant was required to respond. The cue remained on the computer screen for $1000 \mathrm{msec}$ and the subject responded (yes or no) by pressing the appropriate button (index or middle finger) on a response box placed under the right hand. Variable jitter times were inserted before the scenario (0-1500 msec) to enhance the temporal resolution of the BOLD signal. For the baseline rest condition, a blank screen was presented for the duration of the trial. 


\section{METHODS}

\section{Participants}

After excluding two participants due to severe imaging movement artifacts and another two due to poor performance on the behavioral task (less than 65\% correct responses), the final sample included 16 right-handed healthy volunteers ( 9 women; mean age $=26.56$ years; age range $=22-30$ years) with normal or correctedto-normal vision. All participants were native German speakers with no history of neurological or psychiatric illness. None were taking medication at the time of measurement and all gave informed consent before participation. The experimental standards were approved by the local ethics committee of the University of Leipzig in Germany.

\section{Experimental Design}

A $2 \times 2$ factorial design was employed with 48 trials per experimental condition. One factor varied the character type (real or fictional), and the context type (informative or interactive) was varied as the second factor. The experimental conditions, together with a control condition (48 trials) and a resting control baseline condition (24 trials), were presented in a randomized trial design. With a trial length of $8 \mathrm{sec}$ and a total of 264 trials, the experimental session lasted $35.2 \mathrm{~min}$.

The participants were given task instructions and performed a 5-min practice session on a laptop prior to the fMRI session.

\section{MRI Scanning Procedure}

The imaging was carried out on a 3-T Bruker (Ettlingen, Germany) Medspec 30/100 system, which was equipped with the standard birdcage head coil. Participants were placed on the scanner bed in a supine position with their right index and middle fingers positioned on the appropriate response buttons of a two-button response box. The participants' hands were carefully stabilized and formfitting cushions were used to prevent head, arm, and hand movements. Earplugs were also provided to the participants so that scanner noise would be attenuated. The sentences were presented using the VisuaStim Digital MRI Video System (Resonance Technology, Northridge, CA, USA), which is a high-resolution visor $(800 \times 600$ resolution) comprising two small TFT-screens placed close to the eyes.

Twenty-four axial slices $(19.2 \mathrm{~cm}$ field of view; $64 \times$ 64 pixel matrix; $4 \mathrm{~mm}$ thickness; $1 \mathrm{~mm}$ spacing; in-plane resolution of $3 \times 3 \mathrm{~mm}$ ) parallel to bicommissural line (AC-PC) covering the whole brain were acquired using a single-shot gradient echo-planar imaging (EPI) sequence $\left(\mathrm{TR}=2000 \mathrm{msec} ; \mathrm{TE}=30 \mathrm{msec}\right.$; flip angle $=90^{\circ}$; acquisition bandwidth $=100 \mathrm{kHz}$ ) sensitive to blood oxygenation level-dependent (BOLD) contrast. Prior to the functional imaging, 24 anatomical T1-weighted MDEFT images (Lohmann et al., 2001) (data matrix = $256 \times 256$; $\mathrm{TR}=1300 \mathrm{msec} ; \mathrm{TI}=650 \mathrm{msec} ; \mathrm{TE}=10 \mathrm{msec})$ with the same spatial orientation as the functional data were acquired.

\section{fMRI Data Analysis}

The fMRI data were processed using the LIPSIA software package (Lohmann et al., 2001), which contains tools for preprocessing, registration, statistical evaluation, and presentation of fMRI data. Functional data were first motion-corrected using a matching metric on the basis of linear correlation. To correct for the temporal offset between the slices acquired in one scan, a sincinterpolation based on the Nyquist-Shannon Theorem was applied. Low-frequency signal changes and baseline drifts were removed using a temporal high-pass filter with a cutoff frequency of $1 / 96 \mathrm{~Hz}$. Spatial smoothing was performed with a Gaussian filter of $5.65 \mathrm{~mm}$, full width at half maximum.

To align the functional data slices onto a threedimensional stereotactic coordinate reference system, a rigid linear registration was performed with 6 degrees of freedom (3 rotational, 3 translational). The rotational and translational parameters were acquired on the basis of the MDEFT (Norris, 2000; Ugurbil et al., 1993) slices to achieve an optimal match between these slices and the individual three-dimensional reference dataset. This high-resolution, three-dimensional reference dataset was acquired for each subject during a previous scanning session. The MDEFT volume dataset with 160 slices and 1-mm slice thickness was standardized to the Talairach stereotactic space (Talairach \& Tournoux, 1988). These rotational and translational parameters were subsequently normalized in that they were transformed by linear scaling to a Talairach standard size. The normalized parameters were then used to transform the functional slices using trilinear interpolation so that the resulting functional slices were aligned with the stereotactic coordinate system, thus generating output data with a spatial resolution of $3 \times 3 \times 3 \mathrm{~mm}\left(27 \mathrm{~mm}^{3}\right)$. This linear normalization process was enhanced by a subsequent processing step involving an additional nonlinear normalization (Thirion, 1998).

The statistical evaluation was based on a least-squares estimation using the general linear model for serially autocorrelated observations (Friston et al., 1995; Worsley \& Friston, 1995). The design matrix was generated with a box-car function, convolved with the hemodynamic response function. Brain activations were analyzed in an epoch-related design, time-locked to the presentation of the first sentence (the scenario) of all presented trials. The analyzed epoch composed of the duration of the presented scenario (2000 msec). ${ }^{2}$ The model equation, including the observation data, the design matrix, and the error term, was convolved with a Gaussian kernel of dispersion 
of 4 sec, full width at half maximum, to account for the temporal autocorrelation (Worsley \& Friston, 1995). In the following, contrast images or beta value estimates of the raw-score differences between specified conditions were generated for each participant. As all individual functional datasets were aligned to the same stereotactic reference space, the single-subject contrast images were entered into a second-level random-effects analysis for each of the contrasts. One-sample $t$ tests were employed for the group analyses across the contrast images of all subjects, which indicated whether observed differences between conditions were significantly distinct from zero. The $t$ values were subsequently transformed into $Z$ scores.

In addition to direct contrasts, two inclusive masked analyses were also carried out. In one analysis, the statistic parametric map of the random-effects analysis of the real-versus-control direct contrast was used as an inclusive mask in the random-effects analysis of the realversus-fiction direct contrast. In the other analysis, the statistic parametric map of the random-effects analysis of the fiction-versus-control direct contrast was used as an inclusive mask in the random-effects analysis of the fiction-versus-real direct contrast. All the direct contrasts were thresholded on a single voxel level at $p<.001$ prior to the inclusive mask analyses. The findings that result from an inclusive masked analysis indicate which of the brain areas that were significantly activated for Experimental Condition A relative to a Control Condition $\mathrm{C}$ were also significantly activated for Experimental Condition A relative to Experimental Condition B.

Unless indicated otherwise, all reported activations from the direct contrasts and the inclusive masked analyses passed a whole-brain false discovery rate [FDR] (Genovese, Lazar, \& Nichols, 2002; Benjamini \& Hochberg, 1995) threshold of $p<.05$ and a minimum cluster size of 8 voxels $\left(216 \mathrm{~mm}^{3}\right)$.

For the percentage signal change (PSC) analyses of the BOLD response, the mean PSC over the analyzed epoch was extracted from the peak voxel within significantly activated brain areas for the experimental conditions and the resting baseline. The mean PSC of a peak voxel for each condition was calculated in relation to the mean signal intensity of that voxel across time steps.

\section{RESULTS}

\section{Behavioral Data}

Table 1 shows the descriptive data for the behavioral measures. RTs ranged from 872 to $1012 \mathrm{msec}$ and the percentage of correct responses (PCR) across conditions was over $92 \%$. A $2 \times 2$ repeated measures analysis of variance (ANOVA) was carried out on each measure with character type (real vs. fiction) and context type (informative vs. interactive) as the variables. The $2 \times 2$ analyses for RT showed a main effect for both character type $[F(1,15)=38.72, p<.001]$ and context type $[F(1,15)=$
Table 1. Descriptive Data (Mean and Standard Deviation) of the Behavioral Measures (Reaction Time and Percentage of Correct Responses) for All Conditions: Real-informative, Real-interactive, Fiction-informative, Fiction-interactive, and Control

\begin{tabular}{lccccc}
\hline & \multicolumn{2}{c}{$\begin{array}{c}\text { Reaction Time } \\
(\text { msec })\end{array}$} & & Percentage Correct \\
\cline { 2 - 3 } Conditions & Mean & $S D$ & & Mean & $S D$ \\
\hline Real-informative & 877.06 & 169.88 & & 97.92 & 1.86 \\
Real-interactive & 888.81 & 179.88 & & 98.44 & 2.22 \\
Fiction-informative & 913.88 & 165.75 & & 95.18 & 5.2 \\
Fiction-interactive & 1018.06 & 161.65 & & 92.71 & 6.23 \\
Control & 976.19 & 168.32 & & 94.92 & 5.04 \\
\hline
\end{tabular}

28.08, $p<.001]$, as well as a significant interaction effect $[F(1,15)=12.52, p<.01]$. Paired-sample two-tailed $t$ tests showed that this interaction effect resulted from the RT for the fiction-interactive condition being significantly greater than the fiction-informative condition $[t(15)=-4.76, p<.001]$. The difference between the real-interactive and real-informative conditions was nonsignificant $[t(15)=-1.17, p>.1]$.

Paired-samples two-tailed $t$ tests were carried out between each of the four experimental conditions and the control condition. Due to the multiple $t$ test comparisons with the same variable, a Bonferroni correction was applied and the minimum threshold of $p=.0125$ was taken to determine significant effects. The RT for the control condition was significantly longer relative to both the real-informative $[t(15)=-3.88, p<.01]$ and real-interactive conditions $[t(15)=-3.36, p<.01]$.

The $2 \times 2$ repeated measures analyses for PCR showed a main effect for character type $[F(1,15)=27.32, p<$ $.001]$, but no main effect for context type $[F(1,15)=$ $0.715, p>.1]$ nor a significant interaction effect $[F(1$, $15)=1.51, p>.1]$. Paired-samples two-tailed $t$ tests on PCR between the experimental conditions and the control condition revealed only one significant finding-that the percentage correct was lower for the control condition relative to the real-interactive condition $[t(15)=3.51$, $p<.01]$.

In summary, the statistical analyses of the behavioral measures indicated that lengthier RTs and lower PCR were associated with the fiction conditions as well as the control condition relative to the real conditions. Thus, although the fiction and the control conditions were more cognitively demanding relative to the real conditions, they were behaviorally comparable to one another.

\section{fMRI Findings}

To determine which regions of the brain were activated when processing reality testing scenarios, direct con- 
trasts were carried out between the experimental and control conditions. Table 2 shows the list of activations from the activation maps of the real-versus-control and the fiction-versus-control contrast. Overlapping areas of note included the bilateral amygdala and hippocampal formation, the posterior cingulate/retrosplenial cortices (BA 23/29/30), and the dorsal medial prefrontal cortex (PFC) (BA 9) (Figure 2), as well as the superior temporal gyrus, the posterior insula, and the precentral gyrus.

In order to identify the brain regions that were activated when processing scenarios containing either character type, the statistic parametric map of the random-effects analysis of the real/fiction-versus-control contrast was used as an inclusive mask in the randomeffects analysis of the real-versus-fiction and the fictionversus-real contrasts. The reason for these analyses was that the control condition was devised to control for response execution biases (as explained in the Introduction) and possible response selection biases that may arise in association with a particular character type. It could be argued, for instance, that the context need not be fully processed in the case of the real entities because these scenarios are always possible in the real world, whereas the context renders the scenario possible only $50 \%$ of the time with the fictional characters. Thus, higher information processing demands may accompany the latter condition as more information has to be sam- pled in order to make the judgment. In this respect, the control condition is a good match for the fiction conditions as it also requires that two pieces of information be integrated (matching the type of response button and the response value of that button) to select the appropriate response.

To determine which brain regions were activated when processing scenarios containing real entities, the statistic parametric map of the random-effects analysis of the real-versus-control contrast was used as an inclusive mask in the random-effects analysis of the real-versusfiction contrast. Both the direct contrasts were thresholded on a single voxel level at $p<.001$. After correcting for the FDR at a threshold of $p<.05$ and a minimum cluster size of 8 voxels, this inclusive mask analysis revealed significantly activated regions in association with real persons (Table 3a) only in the medial aspect of the anterior PFC $(-2,61,-3)$ and the precuneus/posterior cingulate (PCC: $-2,-50,30)$.

A $2 \times 2$ repeated measures ANOVA was carried out on the mean PSC of the peak voxel in the precuneus/PCC and the anterior PFC to check for interaction effects between character type (real vs. fiction) and context type (informative vs. interactive). The analyses for the precuneus/PCC revealed no main effect for context type $[F(1,15)=0.26, p>.1]$, but showed a significant main effect for character type $[F(1,15)=29.71, p<.001]$ and

Table 2. List of Activations Resulting from the Real-versus-Control Direct Contrast and the Fiction-versus-Control Direct Contrast: Anatomical Specification, Talairach Coordinates $(x, y, z)$, Brodmann's Area (BA), Maximum $Z$ Value, and Volume $\left(\mathrm{mm}^{3}\right)$

\begin{tabular}{|c|c|c|c|c|c|c|c|c|c|c|}
\hline \multirow[b]{2}{*}{ Area } & \multicolumn{5}{|c|}{ Real $>$ Control } & \multicolumn{5}{|c|}{ Fiction $>$ Control } \\
\hline & $x$ & $y$ & $z$ & $m m^{3}$ & $Z$ & $x$ & $y$ & $z$ & $m m^{3}$ & $Z$ \\
\hline Hippocampal formation (left) & -23 & -14 & -12 & 3375 & 5.00 & -20 & -11 & -12 & 6156 & 5.36 \\
\hline Hippocampal formation (right) & 13 & -8 & -15 & 1161 & 4.19 & 19 & -14 & -12 & 1242 & 4.31 \\
\hline Medial prefrontal cortex (BA 9) & -14 & 58 & 30 & 594 & 4.92 & -14 & 58 & 30 & 783 & 4.02 \\
\hline Medial prefrontal cortex (BA 10) & -2 & 61 & -3 & 648 & 4.13 & - & - & - & - & - \\
\hline Precentral gyrus & 28 & -29 & 66 & 216 & 4.26 & 28 & -29 & 66 & 756 & 4.36 \\
\hline Superior temporal gyrus (anterior) & -56 & -8 & -6 & 783 & 3.70 & -56 & -8 & -6 & 810 & 3.63 \\
\hline Superior temporal gyrus (posterior) & - & - & - & - & - & -47 & -35 & 15 & 999 & 3.75 \\
\hline \multirow[t]{2}{*}{ Insula } & 40 & -20 & 18 & 1539 & 3.66 & 37 & -11 & 15 & 4185 & 4.27 \\
\hline & & & & & & 31 & 4 & 12 & 216 & 3.64 \\
\hline \multirow[t]{2}{*}{ Posterior cingulate } & -5 & -53 & 24 & 2106 & 4.30 & -14 & -50 & 6 & 3078 & 4.86 \\
\hline & & & & & & 7 & -53 & 6 & 459 & 3.32 \\
\hline Inferior frontal gyrus (BA 47) & - & - & - & - & - & -41 & 31 & -6 & 324 & 3.43 \\
\hline Occipital cortex (left) & -14 & -98 & 12 & 3132 & 4.60 & -20 & -95 & 9 & 7884 & 4.90 \\
\hline \multirow[t]{2}{*}{ Occipital cortex (right) } & 10 & -89 & 33 & 2241 & 4.04 & 13 & -89 & 15 & 6318 & 4.56 \\
\hline & & & & & & 7 & -74 & -6 & 297 & 3.26 \\
\hline
\end{tabular}

All activations passed an FDR threshold of $p<.05$ and a minimal cluster size of $216 \mathrm{~mm}^{3}$. 
Figure 2. Parallels between activations that resulted from the real-versus-control conditions direct contrast (top) and the fiction-versuscontrol conditions direct contrast (bottom). The images on the left side show activations in for both contrasts in the amygdala and the hippocampal formation $(z=-12)$. The images on the right side show activations in for both contrasts in the PCC and the medial PFC $(z=15)$. The activations passed an FDR threshold of $p<.05$ and a minimum cluster size of 8 voxels.

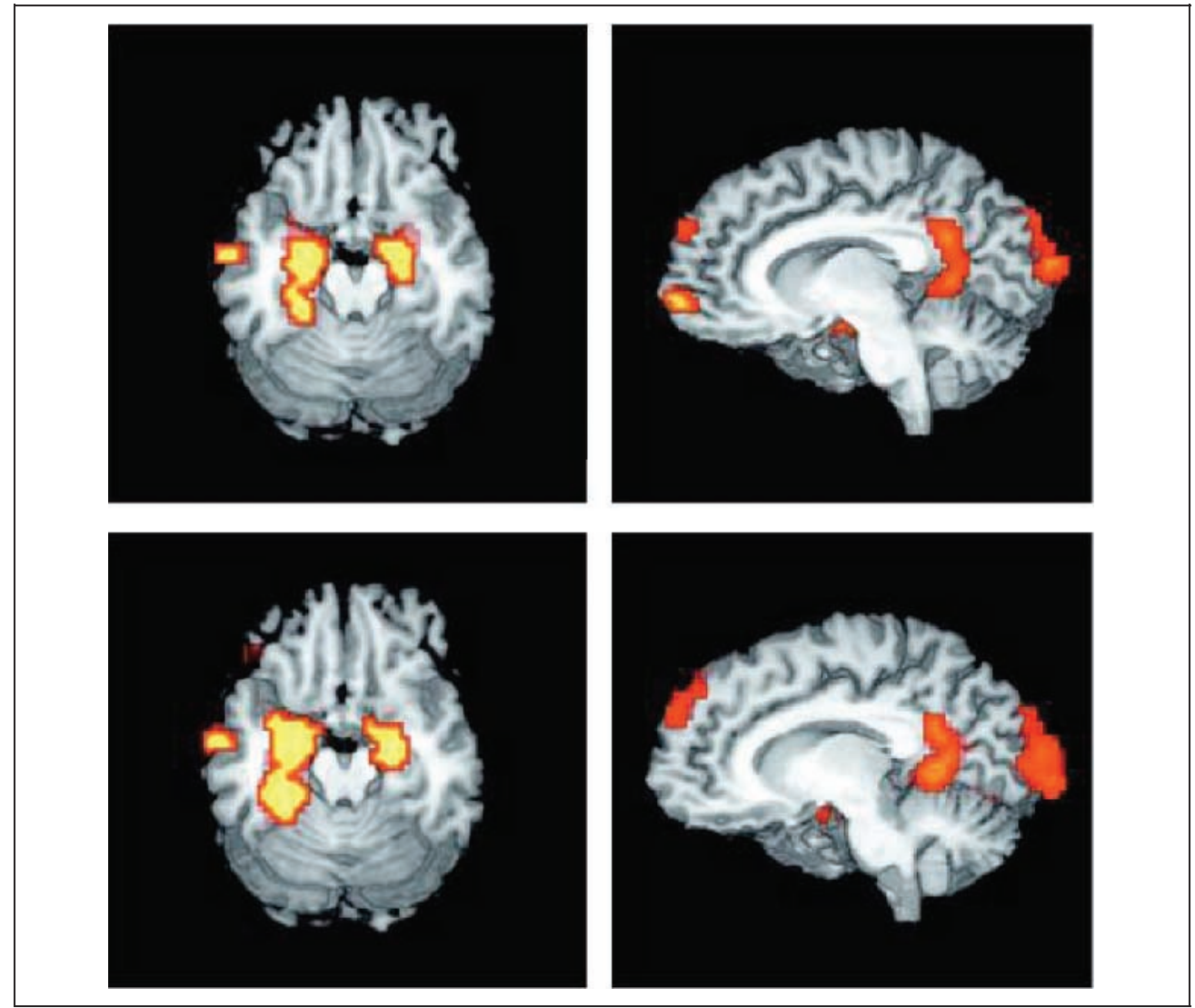

a significant interaction effect $[F(1,15)=5.279, p<.05]$. In the case of the anterior PFC, there was only a significant main effect for character type $[F(1,15)=13.26$, $p<.01]$, but no significant main effect for context type $[F(1,15)=0.89, p>.1]$ nor a significant interaction effect $[F(1,15)=0.04, p>.1]$. Thus, although both the precuneus/PCC and the anterior PFC were more activated when the experimental scenario involved real people in contrast to fictional characters, this differential effect in the precuneus/PCC was significantly more pronounced in the interactive context (Figure 3).

An inclusive mask analysis for the fiction-versus-real contrast, using the fiction-versus-control contrast as a mask (with the same aforementioned parameters), revealed significant activations in two regions in the lateral aspects of the left lateral inferior frontal gyrus (IFG;

Table 3. Anatomical Specification, Talairach Coordinates $(x, y, z)$, Brodmann's Area (BA), Maximum $Z$ Value, and Volume $\left(\mathrm{mm}^{3}\right)$ of the Significantly Activated Areas from the Following Contrasts

\begin{tabular}{|c|c|c|c|c|c|c|}
\hline Area & $x$ & $y$ & $z$ & $B A$ & $m m^{3}$ & $Z-\max$ \\
\hline \multicolumn{7}{|c|}{$\begin{array}{l}\text { (a) Processing of Real Persons Relative to Fictional Characters and the Control Condition: Real > Fiction Contrast (Inclusive } \\
\text { Mask: Real > Control) }\end{array}$} \\
\hline Anterior medial PFC & -2 & 61 & -3 & 10 & 621 & 3.81 \\
\hline Precuneus/posterior cingulate & -2 & -50 & 30 & $23 / 31$ & 405 & 4.52 \\
\hline \multicolumn{7}{|c|}{$\begin{array}{l}\text { (b) Processing of Fictional Characters Relative to Real Persons and the Control Condition: Fiction > Real Contrast (Inclusive } \\
\text { Mask: Fiction > Control) }\end{array}$} \\
\hline Inferior frontal gyrus & -41 & 28 & 6 & 45 & 351 & 5.39 \\
\hline Inferior frontal gyrus & -35 & 31 & -3 & 47 & 810 & 4.95 \\
\hline Medial occipital gyrus & -26 & -95 & 3 & 18 & 2187 & 5.17 \\
\hline
\end{tabular}

All activations passed a False Discovery Rate threshold of $p<.05$ and a minimal cluster size of $216 \mathrm{~mm}^{3}$. 
Figure 3. Processing of real conditions (inclusive mask analysis of the real $>$ fiction contrast using the real $>$ control contrast as a mask). Contrast maps and mean percentage signal change plots for regions in the (top) anterior medial PFC (BA 10: $-2,61,-3)$ and (bottom) precuneus/PCC (BA 23/31: $-2,-50,30)$, which showed significantly greater activation during the processing of real conditions relative to fiction conditions. The graphs display the mean and standard error of the mean for all conditions and the zero point in the graphs represents the resting baseline. All reported activations passed an FDR threshold of $p<.05$ and a minimum cluster size of 8 voxels.
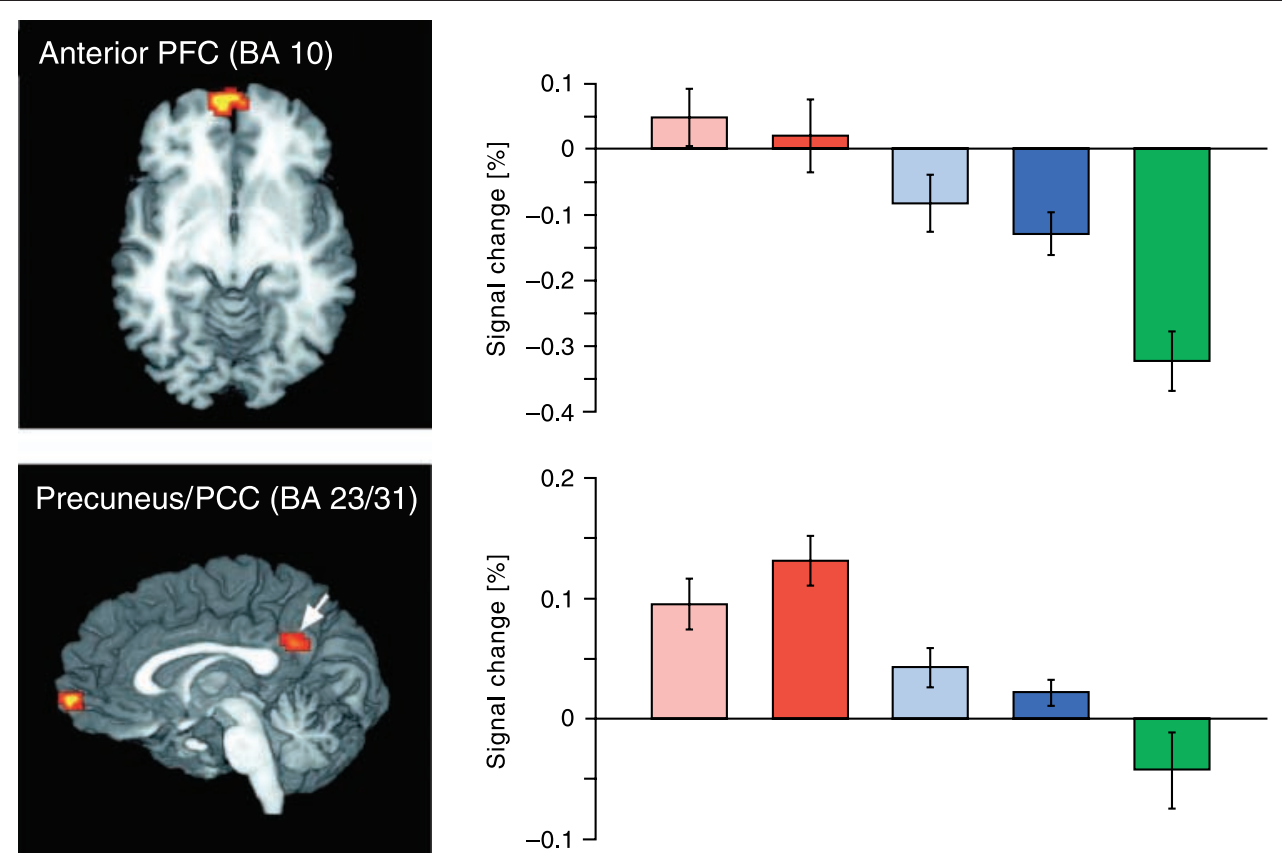

real-informative

fiction-informative

control
Figure 4. Processing of fiction conditions (inclusive mask analysis of the fiction $>$ real contrast using the fiction $>$ control contrast as a mask). Contrast maps (sagittal and coronal views) for two regions in the left lateral IFG: BA 47 $(-35,31,-3)$ and BA $45(-41$, $28,6)$. Both regions showed significantly greater activation during the processing of fiction conditions relative to real and control conditions. The graphs display the mean and standard error of the mean for all conditions and the zero point in the graphs represents the resting baseline. All reported activations passed an FDR threshold of $p<.05$ and a minimum cluster size of 8 voxels.
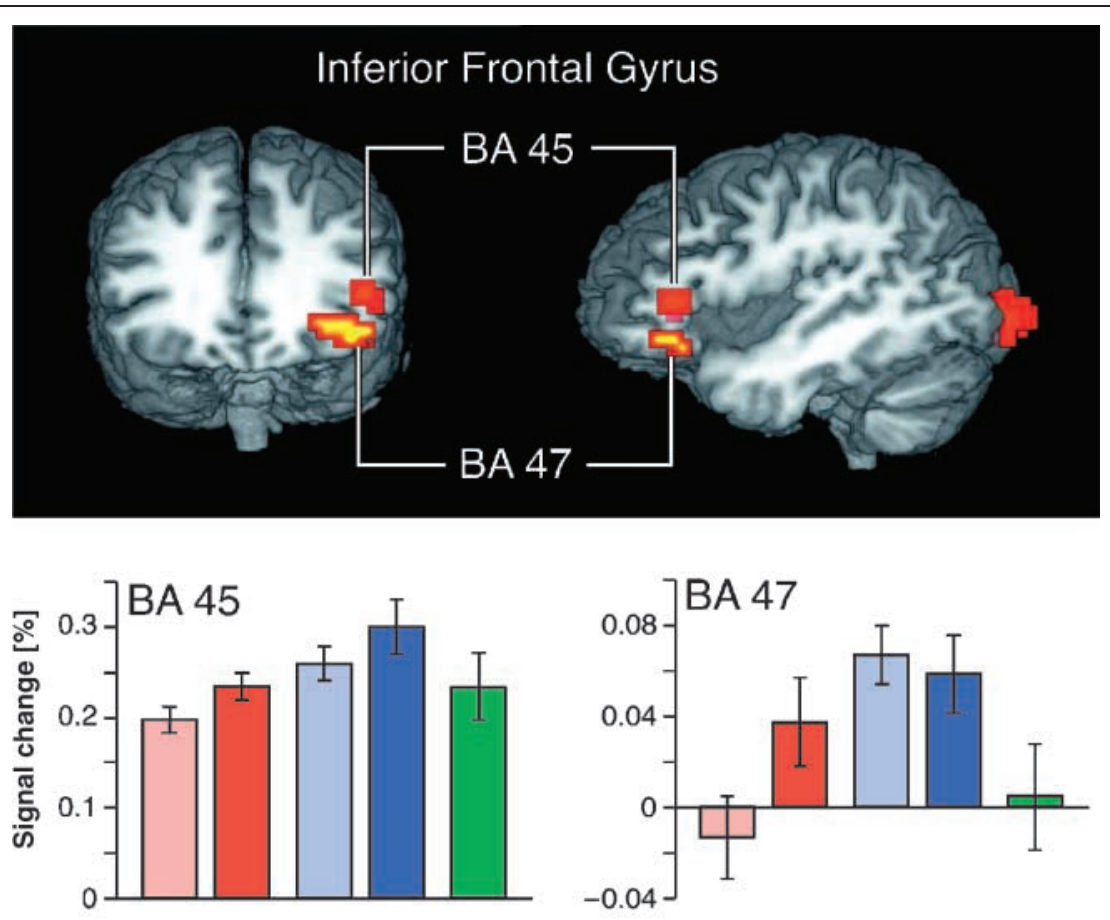

real-informative

fiction-informative control real-interactive fiction-interactive 
BA 45 and BA 47) associated with the processing of contexts containing fictional characters (Figure 4; Table 3b).

A repeated measures ANOVA was carried out on the mean PSC of the peak voxel in both IFG regions to check for interaction effects between character type (real vs. fiction) and context type (informative vs. interactive). The BA 45 region revealed a main effect for character type $[F(1,15)=8.86, p<.01]$ and context type $[F(1,15)=$ $5.56, p<.05]$, whereas the BA 47 region showed a significant main effect for character type $[F(1,15)=14.88$, $p<.01]$ but not for context type $[F(1,15)=1.43, p>.1]$. There were no significant interaction effects found for either the BA $45[F(1,15)=0.37, p>.1]$ or the BA 47 $[F(1,15)=2.35, p>.1]$ regions.

As a matter of interest, direct (unmasked) contrasts of the experimental conditions (minimum volume of $216 \mathrm{~mm}^{3}$ and an uncorrected threshold of $Z>3.09$ at the voxel level) were also carried out. In the case of activations that arise when processing contexts containing real entities relative to contexts containing fictional characters, significantly activated areas included the anterior $\mathrm{MPFC}$ and the PCC/precuneus as well as parts of the somatosensory cortex. In the case of activations associated with processing contexts containing fictional characters relative to contexts containing fictional entities, significantly activated areas included not only the areas along the lateral IFG seen in the masked contrast but also more extensive activations over a wide range of other areas, including the bilateral lateral middle and inferior frontal gyri, the posterior medial PFC (BA 8/6), the inferior parietal lobule, the thalamus, and the caudate nucleus. This pattern for the fiction $>$ real direct contrast was, in fact, quite similar to that found in the control $>$ real/fiction direct contrasts (not reported here). Thus, making judgments within the fiction conditions compared to the real condition was generally associated with higher information processing demands, as would be expected given the behavioral findings. These effects were, however, highest in the control condition.

Although using inclusive masked analyses or direct contrasts did not make much difference in the case of findings associated with the real entities, this was a pertinent issue in the case of the fiction conditions. For the sake of consistency in reporting results and the rationale of our experimental design, we only concentrate on the results of the inclusive mask analyses. As mentioned earlier, our objective when employing the control condition was to be able to tease apart the areas that were engaged due to differences in processing demands (and possibly other unrelated processes) from those that were directly relevant to the central questions posed by the study. The masked contrasts allowed us to be able to focus on the few areas that were found to be associated with processing contexts containing fictional characters (or real entities) relative to contexts containing real entities (or fictional characters) and a high demand unrelated context.

\section{DISCUSSION}

The processing of scenarios containing real or fictional characters resulted in activations of overlapping brain regions when contrasted with the control condition. Of chief interest are the common activations of the bilateral hippocampal formation and amygdala, which were associated with both the experimental conditions relative to the control condition. Medial-temporal structures, such as the hippocampal formation, are known to be crucial for declarative memory or the conscious memory of facts (or semantic memory) and events (or episodic memory) (Eichenbaum, 2004; Squire, Stark, \& Clark, 2004). Also noteworthy were the activations in the dorsal aspect of the medial PFC (BA 9) and the posterior cingulate and retrosplenial regions, areas that have been routinely implicated in tasks requiring mental state reasoning (Frith \& Frith, 2006; Saxe, 2006). The largely overlapping activation patterns between the areas implicated when processing scenarios containing real or fictional characters is indicative of the similarity of the underlying processes involved when engaging in these scenarios. What we went on to explore were the finer distinctions when processing of contexts containing fictional and real characters so as to attain a better understanding of how we understand the distinction between reality and fiction.

\section{Real Entities}

With regard to selective responses associated with character type information, the anterior PFC (medial BA 10) and the precuneus/PCC were found to be selectively engaged during the processing of contexts containing real entities relative to contexts containing fictional entities. Both these areas belong to the network of structures that show the highest metabolic activity in comparison to other brain regions during rest (Raichle et al., 2001), and are commonly implicated in tasks that involve evaluative judgment as well as episodic memory retrieval and self-referential processing (Cavanna \& Trimble, 2006; Gilbert et al., 2006; Wagner, Shannon, Kahn, \& Buckner, 2005; Ramnani \& Owen, 2004).

As the anterior PFC has been found to play a role in several other processes including processing of internal states, prospective memory, source memory, cognitive branching, and relational integration, Ramnani and Owen (2004) proposed that this area is involved when a higherorder behavioral goal requires the integration of information from two or more separate cognitive operations. At first glance, our findings do not appear to lend support to this idea because the same amount of stimuli and types of contexts were processed in all experimental conditions, and the activation difference is thus only a function of the type of character representation.

One of the major differences between the real entities, such as the famous persons, and fictional characters is the amount of information that we can readily draw 
upon in reference to each character type. Although we can arrive at quite a rich understanding of a fictional world (such as that of Harry Potter), we still have, relatively speaking, limited information about his world in comparison to what we know about our own world.

With an entity such as George Bush, one has access to different types of information about him: the way he looks, his position in the social hierarchy, the degree of influence his politics has on one's own life, what values he stands for, one's personal feelings toward him, the last time one saw him on television, and so on. It is therefore possible that the anterior PFC activation here reflects the integration and coordination of many kinds of information, including episodic and self-referential ones, which are automatically accessed with the introduction of a familiar entity, even when there is no externally directed explicit behavioral goal that imposes such demands. In fact, the role played by medial prefrontal and medial parietal cortices (in conjunction with medial-temporal lobe regions) in anticipatory associative processing has been recently highlighted in a proposal on the proactive nature of the brain (Bar, 2007).

The role of the precuneus/PCC also fits in with this rationale because, like the anterior $\mathrm{PFC}$, it also plays a key role in episodic memory retrieval and self-processing operations (Cavanna \& Trimble, 2006; Vogt \& Laureys, 2005; Wagner et al., 2005). On the one hand, as the participants had not met all the famous people they were introduced to in the scenario, episodic memories stemming from actual encounters are lacking. However, because we occupy the same world as famous people, information about them is generally far more socially relevant for us when compared to fictional characters. In fact, a recent study has demonstrated that simply viewing social interactions between real people relative to identical scenarios performed by animated agents results in higher activations in the PCC/ precuneus (Mar, Kelley, Heatherton, \& Macrae, 2007).

As a consequence then, information concerning real entities is more personally significant or self-relevant, and this facet could account for why they are more "episodic" in nature. William James argued on similar grounds with the statement, "In the relative sense, then, the sense in which we contrast reality with simple unreality, ... reality means simply relation to our emotional and active life" (James, 1950, p. 295, original italics).

The interaction effect showing the highest activation in the precuneus/PCC for the real-interactive condition is also noteworthy in this context of the responsiveness of the precuneus to potentially self-relevant information (Cavanna \& Trimble, 2006; Northoff et al., 2006; Vogt \& Laureys, 2005), as possible interactions with real people are, of course, more socially relevant for oneself than possible interactions with fictional characters. Some indirect support for this idea comes from an fMRI study that employed interactive games such as the Prisoner's Dilemma Game and the Ultimatum Game (Rilling, Sanfey, Aronson, Nystrom, \& Cohen, 2004). Higher PCC/precuneus
(BA 7/31) activations were reported when participants played against human partners relative to computer partners, and the authors interpreted this finding in terms of more personally meaningful consequences arising in the former situation as it involved real social interactions (Rilling et al., 2004).

It has even been suggested that episodic memory of the past is crucial for more generative open-ended processes beyond memory retrieval (Buckner \& Carroll, 2007; Tulving, 1983, 2005), such as episodic prospection or the ability to imagine oneself in the possible future contexts (Addis, Wong, \& Schacter, 2007; Szpunar, Watson, \& McDermott, 2007), mentalizing (Saxe \& Kanwisher, 2003 as cited in Buckner \& Carroll, 2007), and navigation, all of which involve self-projection (Buckner \& Carroll, 2007) or engaging in alternative perspectives (for a competing interpretation, see Hassabis \& Maguire, 2007). What is interesting is that our findings suggest that some of the brain areas underlying such abilities are more strongly implicated even when thinking about possible events with reference to real entities as opposed to fictional characters.

Related findings have been reported by Szpunar et al. (2007), where they found greater engagement of similar brain regions when imagining themselves in a specific future or past situation compared to when imagining a familiar entity, Bill Clinton, participating in such events. Also, Hassabis and Maguire (2007) reported unpublished findings in their recent review where they found that these cortical midline structures were preferentially engaged when recalling real episodic memories as opposed to recalling imaginary memories. Unlike in the present study, where no explicit episodic memories were required to be processed, what was manipulated within both the aforementioned studies was a relative difference of episodic information and associated self-relevance in question. It appears then that the more self-relevant and familiar the information, the higher the activation in the anterior medial PFC as well as the precuneus and posterior cingulate cortices.

In fact, a proposal concerning the distinction between the functions of the dorsal and ventral medial PFC as underlying two different top-down systems in the appraisal of self-relevance has been recently put forward (Schmitz \& Johnson, 2007). The ventral system is said to "mediate identification and appraisal of stimulus-induced self-relevance," whereas the dorsal system mediates "cognitive control in the generation of explicitly self-referential decisions." If we were to interpret the current findings using this framework, we could postulate that the involvement of the dorsal medial PFC system for representations containing both real and fictional characters relative to the control task suggests undifferentiated involvement of the cognitive control processes of evaluation, introspection, and recollection for both. The preferential engagement of the ventral medial PFC when processing contexts containing real entities, on the other hand, speaks for the possibility that this is because such information is, 
relatively speaking, more salient and self-relevant compared to contexts containing fictional characters.

\section{Fictional Characters}

Quite a different pattern of results emerged when assessing the neural response associated with processing contexts containing fictional entities. Two areas (BA 47, BA 45) along the left lateral IFG were selectively activated during the fiction conditions. Both of these IFG areas have been consistently reported to play a key role in semantic aspects of language processing (Hagoort, 2005; Bookheimer, 2002; Martin \& Chao, 2001). In fact, similar regions in the IFG were found to be activated as a response to both semantic violations and world knowledge violations in contrast to statements that were correct on semantic and world knowledge grounds (Hagoort, Hald, Bastiaansen, \& Petersson, 2004).

BA 47, in particular, is held to be crucial for executive facets of semantic information processing that are called upon when comparing or making judgments about the semantic relationships between representations held in working memory (Bookheimer, 2002). Badre, Poldrack, Pare-Blagoev, Insler, and Wagner (2005) have, in fact, argued for a dissociation of the semantic functions of these two regions in proposing that the mid-IFG (BA 45) underlies selection between competing alternatives, whereas the anterior IFG (BA 47) is sensitive to the associative strength of alternatives and is involved in situations that require controlled semantic retrieval.

Regarding the implications of these findings for the present study, although the chosen fictional entities were very familiar to the participants, access to information concerning these entities was likely to be less dominantly accessible than in the case of the famous individuals. The weaker, less automatic, or nonroutine access to semantic knowledge concerning these fictional entities, as well as to the necessary related information to make the judgment for the task at hand, could explain the higher activation in such semantic selection and retrieval areas.

It is important to note that this apparent dissociation between the processing of fiction (compared to reality) as factual knowledge in contrast to reality (compared to fiction) as subjectively coded representations is to be regarded in relative terms. If the degree of coded self-relevance of a representation is one of the factors that affects what we take to be real and unreal, it may be necessary to approach the question of how we process fiction versus reality not simply in terms of a dichotomy (between what is classified as universally real and unreal), but in terms of the degree of personal relevance associated with the characters in question. Such a continuum-based approach may be crucial to fully understand various fascinating aspects of human behavior, including religiosity and chronic gaming. In such contexts, the reality/fiction distinction can be fuzzy given that these fictional contexts tend to be coded in highly self-relevant terms for some people.

\section{Alternative Interpretations}

One alternative interpretation for the patterns of activation to the ones we have provided is that the difficulty difference between the real and famous conditions may instead explain the findings. The involvement of default state areas in the real conditions could reflect easier task demands in these conditions, whereas the lateral PFC activations in the fiction conditions could be attributed to greater executive function demands. We believe that these interpretations do not adequately account for the results for the following reasons.

Firstly, we carried out masked contrasts with a demanding control condition, which was well-matched to the fiction conditions, so that components related to difficulty and greater effort could be subtracted from the patterns of activations seen in the experimental conditions. In fact, the direct contrasts of the control condition with both experimental conditions (not reported here) reflect recruitment of areas classically associated with task difficulty, uncertainty, cognitive effort, working memory, and higher executive control demands such as dorsal aspects of the lateral PFC (encompassing BA 9/ 46), medial aspects of the posterior PFC (BA 8/6), and subcortical structures (Graybiel, 2005; Volz, Schubotz, \& von Cramon, 2005; Passingham \& Sakai, 2004; Carpenter, Just, \& Reichle, 2000). The fiction conditions were instead selectively associated with activations of ventral aspects of the lateral PFC (BA 45/47), areas that are selective for semantic processing, and not unspecific difficulty demands.

Secondly, the argument that activation of the anterior medial PFC and the precuneus/PCC reflects that subjects have to be less engaged in the real conditions can also be rejected. If this supposition were correct, the pattern of activations associated with these regions should be characterized by smaller deactivations below baseline in the real conditions compared to larger deactivations below baseline in the fiction conditions, because activations during rest should lead to the strongest activations in regions that are part of the default mode network. The data we report for the real conditions, however, show activations above those of the resting baseline (see bar graphs in Figure 3). The findings thus point to a stronger engagement of the processes that are subserved by these regions when faced with scenarios involving real entities.

\section{Conclusions}

In summary, the findings of the present study essentially imply that different memory retrieval systems are more strongly recruited when making reality-based judgments about scenarios involving real or fictional characters. 
Although both scenario types recruited neural areas associated with declarative memory processes such medialtemporal lobe structures, contexts containing fictional entities also significantly activated more semantically relevant retrieval areas, whereas those containing real people significantly engaged more episodically relevant retrieval regions. It appears then that one of the means by which we tell reality apart from fiction, at least in the explicit context of reality testing, seems to lie in the manner in which such information is coded and accessed, namely, if it is personally significant or not. The degree of associated self-relevance is therefore a possibly critical determinant factor that enables us to differentiate between what is real and unreal.

\section{Acknowledgments}

We thank Uta Wolfensteller, Andreja Bubic, Dirk Schulze, and the two anonymous reviewers for their valuable feedback on the manuscript. We are also grateful to Andrea Gast-Sandmann for her assistance with the figures, Bettina Johst for her help with the programming of the experiment, and Stefan Zysset for his advice concerning the fMRI data preprocessing and analyses.

Reprint requests should be sent to Anna Abraham, Department of Cognitive Neurology, Max Planck Institute for Human Cognitive and Brain Sciences, Stephanstr. 1a, D-04103 Leipzig, Germany, or via e-mail: abraham@cbs.mpg.de.

\section{Notes}

1. It is to be noted that people do confuse reality with fantasy in other contexts outside of ongoing experience, as evident from source or reality monitoring errors that often surface when recalling memories.

2. This time point was selected due to the fact that the subjects anticipatorily make the impending judgment concerning the possibility (or truth) of the scenario while reading the statement itself (as evident from their feedback). The question cue that follows acts as a prompt which indicates how their response should be shaped (left or right button press) based on the cue that follows. Thus, although the overt decision indicating the possibility (or truth) or the scenario is only made later, the possibility (or truth) assessment was made in advance.

\section{REFERENCES}

Addis, D. R., Wong, A. T., \& Schacter, D. L. (2007). Remembering the past and imagining the future: Common and distinct neural substrates during event construction and elaboration. Neuropsychologia, 45, 1363-1377.

Badre, D., Poldrack, R. A., Pare-Blagoev, E. J., Insler, R. Z., \& Wagner, A. D. (2005). Dissociable controlled retrieval and generalized selection mechanisms in ventrolateral prefrontal cortex. Neuron, 47, 907-918.

Bar, M. (2007). The proactive brain: Using analogies and associations to generate predictions. Trends in Cognitive Sciences, 11, 280-289.

Benjamini, Y., \& Hochberg, Y. (1995). Controlling the false discovery rate: A practical and powerful approach to multiple testing. Journal of the Royal Statistical Society: Series B, 57, 289-300.
Bookheimer, S. (2002). Functional MRI of language: New approaches to understanding the cortical organization of semantic processing. Annual Review of Neuroscience, 25, 151-188.

Buckner, R. L., \& Carroll, D. C. (2007). Self-projection and the brain. Trends in Cognitive Sciences, 11, 49-57.

Carpenter, P. A., Just, M. A., \& Reichle, E. D. (2000). Working memory and executive function: Evidence from neuroimaging. Current Opinion in Neurobiology, 10, 195-199.

Cavanna, A. E., \& Trimble, M. R. (2006). The precuneus: A review of its functional anatomy and behavioural correlates. Brain, 129, 564-583.

Eichenbaum, H. (2004). Hippocampus: Cognitive processes and neural representations that underlie declarative memory. Neuron, 44, 109-120.

Friston, K., Holmes, A., Worsley, K., Poline, J., Frith, C., \& Frackowiak, R. (1995). Statistical parametric maps in functional imaging: A general linear approach. Human Brain Mapping, 2, 189-210.

Frith, C. D., \& Frith, U. (2006). The neural basis of mentalizing. Neuron, 50, 531-534.

Genovese, C. R., Lazar, N. A., \& Nichols, T. (2002). Thresholding of statistical maps in functional neuroimaging using the false discovery rate. Neuroimage, 15, 870-878.

Gerrig, R. J. (1993). Experiencing narrative worlds. New Haven, CT: Yale University Press.

Gilbert, S. J., Spengler, S., Simons, J. S., Steele, J. D., Lawrie, S. M., Frith, C. D., et al. (2006). Functional specialization within rostral prefrontal cortex (area 10): A meta-analysis. Journal of Cognitive Neuroscience, 18, 932-948.

Graybiel, A. M. (2005). The basal ganglia: Learning new tricks and loving it. Current Opinion in Neurobiology, 15, 638-644.

Green, M. C., \& Brock, T. C. (2000). The role of transportation in the persuasiveness of public narratives. Journal of Personality and Social Psychology, 79, 701-721.

Green, M. C., Brock, T. C., \& Kaufman, G. F. (2004). Understanding media enjoyment: The role of transportation into narrative worlds. Communication Theory, 14, 311-327.

Hagoort, P. (2005). On Broca, brain, and binding: A new framework. Trends in Cognitive Sciences, 9, 416-423.

Hagoort, P., Hald, L., Bastiaansen, M., \& Petersson, K. M. (2004). Integration of word meaning and world knowledge in language comprehension. Science, 304, 438-441.

Hassabis, D., \& Maguire, E. A. (2007). Deconstructing episodic memory with construction. Trends in Cognitive Sciences, 11, 299-306.

James, W. (1950). The principles of psychology (Vol. 2). New York: Dover Publications.

Lohmann, G., Muller, K., Bosch, V., Mentzel, H., Hessler, S., Chen, L., et al. (2001). LIPSIA-A new software system for the evaluation of functional magnetic resonance images of the human brain. Computerized Medical Imaging and Graphics, 25, 449-457.

Mar, R. A. (2004). The neuropsychology of narrative: Story comprehension, story production and their interrelation. Neuropsychologia, 42, 1414-1434.

Mar, R. A., Kelley, W. M., Heatherton, T. F., \& Macrae, C. N. (2007). Detecting agency from the biological motion of veridical $v s$ animated agents. Social Cognitive and Affective Neuroscience, 2, 199-205.

Marsh, E. J., Meade, M. L., \& Roediger, H. L., III (2003). Learning facts from fiction. Journal of Memory and Language, 49, 519-536.

Martin, A., \& Chao, L. L. (2001). Semantic memory and the brain: Structure and processes. Current Opinion in Neurobiology, 11, 194-201. 
Nell, V. (1988). Lost in a book: The psychology of reading for pleasure. Mahwah, NJ: Yale University Press.

Nell, V. (2002). Mythic structures in narrative: The domestication of immortality. In M. C. Green, J. J. Strange, \& T. C. Brock (Eds.), Narrative impact: Social and cognitive foundations (pp. 17-37). Mahwah, NJ: Erlbaum.

Norris, D. G. (2000). Reduced power multislice MDEFT imaging. Journal of Magnetic Resonance Imaging, 11, 445-451.

Northoff, G., Heinzel, A., de Greck, M., Bermpohl, F., Dobrowolny, H., \& Panksepp, J. (2006). Self-referential processing in our brain - A meta-analysis of imaging studies on the self. Neuroimage, 31, 440-457.

Oatley, K. (2002). Emotions and the story worlds of fiction. In M. C. Green, J. J. Strange, \& T. C. Brock (Eds.), Narrative impact: Social and cognitive foundations (pp. 39-69). Mahwah, NJ: Erlbaum.

Passingham, D., \& Sakai, K. (2004). The prefrontal cortex and working memory: Physiology and brain imaging. Current Opinion in Neurobiology, 14, 163-168.

Raichle, M. E., MacLeod, A. M., Snyder, A. Z., Powers, W. J., Gusnard, D. A., \& Shulman, G. L. (2001). A default mode of brain function. Proceedings of the National Academy of Sciences, U.S.A., 98, 676-682.

Ramnani, N., \& Owen, A. M. (2004). Anterior prefrontal cortex: Insights into function from anatomy and neuroimaging. Nature Reviews Neuroscience, 5, 184-194.

Rilling, J. K., Sanfey, A. G., Aronson, J. A., Nystrom, L. E., \& Cohen, J. D. (2004). The neural correlates of theory of mind within interpersonal interactions. Neuroimage, 22, 1694-1703.

Saxe, R. (2006). Uniquely human social cognition. Current Opinion in Neurobiology, 16, 235-239.

Saxe, R., \& Kanwisher, N. (2003). People thinking about thinking people. The role of the temporo-parietal junction in "theory of mind". Neuroimage, 19, 1835-1842.

Schmitz, T. W., \& Johnson, S. C. (2007). Relevance to self: A brief review and framework of neural systems underlying appraisal. Neuroscience and Biobehavioral Reviews, 31, $585-596$.
Skolnick, D., \& Bloom, P. (2006). What does Batman think about SpongeBob? Children's understanding of the fantasy/fantasy distinction. Cognition, 101, B9-B18.

Squire, L. R., Stark, C. E., \& Clark, R. E. (2004). The medial temporal lobe. Annual Review of Neuroscience, 27, 279-306.

Szpunar, K. K., Watson, J. M., \& McDermott, K. B. (2007). Neural substrates of envisioning the future. Proceedings of the National Academy of Sciences, U.S.A., 104, 642-647.

Talairach, J., \& Tournoux, P. (1988). Co-planar stereotaxic atlas of the human brain. New York: Thieme.

Thirion, J. P. (1998). Image matching as a diffusion process: An analogy with Maxwell's demons. Medical Image Analysis, 2, 243-260.

Tulving, E. (1983). Elements of episodic memory. Oxford: Oxford University Press.

Tulving, E. (2005). Episodic memory and autonoesis: Uniquely human? In H. S. Terrace \& J. Metcalfe (Eds.), The missing link in cognition: Origins of self-reflective consciousness. Oxford: Oxford University Press.

Ugurbil, K., Garwood, M., Ellermann, J., Hendrich, K., Hinke, R., Hu, X., et al. (1993). Imaging at high magnetic fields: Initial experiences at 4 T. Magnetic Resonance Quarterly, 9, 259-277.

Vogt, B. A., \& Laureys, S. (2005). Posterior cingulate, precuneal and retrosplenial cortices: Cytology and components of the neural network correlates of consciousness. Progress in Brain Research, 150, 205-217.

Volz, K. G., Schubotz, R. I., \& von Cramon, D. Y. (2005). Variants of uncertainty in decision-making and their neural correlates. Brain Research Bulletin, 67, 403-412.

Wagner, A. D., Shannon, B. J., Kahn, I., \& Buckner, R. L. (2005). Parietal lobe contributions to episodic memory retrieval. Trends in Cognitive Sciences, 9, 445-453.

Woolley, J. D., \& Wellman, H. M. (1990). Young children's understanding of realities, nonrealities, and appearances. Child Development, 61, 946-961.

Worsley, K. J., \& Friston, K. J. (1995). Analysis of fMRI time-series revisited-Again. Neuroimage, 2, 173-181. 